\title{
Modeling College Admission as a Signaling Game
}

\author{
Joseph Rekus ${ }^{1}, \&$ Yixiao Jiang ${ }^{1}$ \\ ${ }^{1}$ Christopher Newport University, United States \\ Correspondence: Yixiao Jiang, Department of Economics, Christopher Newport University, United States.
}

Address: 1 Avenue of the Arts, Newport News, VA 23606, USA.

Received: September 16, 2021

Accepted: October 15, 2021

Available online: October 26, 2021

doi:10.11114/aef.v8i6.5394

URL: https://doi.org/10.11114/aef.v8i6.5394

\begin{abstract}
This paper models the college admission process as a signaling game between the admissions office and a pool of heterogeneous applicants characterized by academic abilities and demonstrated interest. In the screening and selection process, applicants attempt to signal their private information through their performance on a standardized test (for academic abilities) and interview (for demonstrated interest). We show, under general conditions, that a separating equilibrium exists in which it is incentive-compatible for applicants to reveal their characteristics truthfully, and thus the admissions office does not fall victim to the problem of information asymmetry. Furthermore, we delineate how this equilibrium can be induced by setting appropriate "scoring" thresholds associated with standardized tests and interviews.
\end{abstract}

Keywords: asymmetry of information, college admission, signaling game, separating equilibrium

\section{Introduction}

In regard to the distribution of scholarship awards, it is a common strategy for university admissions offices to first stratify the prospective students into categories based on a selected set of metrics (such as high or low academic ability, initiative, etc.), then allocate the awards among the groups in a hierarchical manner. While this is an intuitive and widely used strategy, it has some basic complications which stem from the asymmetry of information: that is, the admissions office's lack of an exact knowledge of the students' caliber. Consequently, applicants have the incentive to imitate the behavior of other applicants who are more suited to receive larger scholarship awards. An example of such imitation would be lower-level students taking on substantial costs to score higher on standardized tests than they otherwise would have.

The general problem of information asymmetry (and how to overcome it) has been widely studied in economics. In the labor market, employees often have better knowledge of their own ability than employers. According to Spence (1973), education credentials can be used as a signal to employers, indicating a higher level of ability that the potential employee may possess; thereby narrowing the information gap. Similar problems have been analyzed in the context of the insurance market (Rothschild \& Stiglitz, 1976), strategic disclosure of accounting information (Bushman \& Smith, 2001; Ball, Robin \& Wu, 2003), corporate governance (Farber, 2005), and many others. To the best of our knowledge, the information asymmetry between the admissions office and college applicants has not been studied in a formal economic model when the former is distributing a scholarship.

When the problem of information asymmetry goes unaddressed admissions offices get tricked into inefficiently allocating their scholarship awards. This leads to several unwanted effects, including the "crowding-out" of higher-level students and the overspending on students of lower caliber. According to Bound, Hershbein, and Long (2009), students have increasingly seen an incentive to amplify competitive behavior, particularly when it comes to standardized testing. There has also been an increase in the percentage of students seeking formal help in standardized test preparation and that access to this type of assistance has become more available, particularly for students of high-income families.

All of this seemingly suggests the obvious: in general, imitative behavior is a prevalent phenomenon in the college admissions process and increasingly so. But what is even more disturbing is that Bound et al. (2009) had not found any evidence that an intensification of test-taking and test-prep culture has led to a general increase in academic ability. This suggests that imitative behavior also imposes an economic cost on applicants and hence gives more reason for the admissions office to combat it. 
We consider the strategic interaction between the admissions office and a pool of applicants with incomplete information: only applicants themselves know their academic ability and interest in the school. The admissions office uses standard test score and an interview to screen and select candidates for scholarship. The contribution of this paper is to develop an incentive-compatible mechanism that prompts students to truthfully reveal their private information. Built on the seminal work of Spence (1973), we rigorously derive conditions that induce a separating equilibrium in which applicants of different types send different signals to the admissions office. We will also use our analysis to then make recommendations to admissions offices regarding admissions strategy.

The rest of this paper is organized as follow. Section 2 describes the model in a general set up. Section 3 solves for the equilibrium. Section 4 provides an illustration based on a convenient cost function. Section 5 discusses comparative statics. Section 6 discusses implementation of the model. Section 7 concludes.

\section{The Model}

We seek to model the strategic interaction of two players: a representative college applicant and the university's admissions office. As yield becomes a growingly important concern for universities (Clinedinst, 2019), the admissions office uses metrics like the level of contacts to identify students who are more likely to accept their offers (Deardon, Li, Meyerhoefer \& Yang 2017). To reflect this, admission and scholarship decisions in our model depend not only on applicants' academic ability but also their demonstrated interests. To be specific, the applicant (which we will also refer to as $P I)$ is characterized by a vector $\left(\theta^{A}, \theta^{D}\right)$ representing his/her academic ability and demonstrated interest. Each trait can be characterized by "high" or "low." Hence, as displayed in Table 1 below, the pool of applicants consists of four types,

Table 1. Types of students (P1).

\begin{tabular}{|l|c|c|}
\hline Types of Students (P1) & High Ability & Low Ability \\
\hline High Demonstrated interest & Type 1: $\left(\boldsymbol{\theta}_{H}^{\boldsymbol{A}}, \boldsymbol{\theta}_{H}^{\boldsymbol{D}}\right)$ & Type 2: $\left(\boldsymbol{\theta}_{L}^{\boldsymbol{A}}, \boldsymbol{\theta}_{H}^{\boldsymbol{D}}\right)$ \\
\hline Low Demonstrated interest & Type 3: $\left(\boldsymbol{\theta}_{H}^{\boldsymbol{A}}, \boldsymbol{\theta}_{L}^{\boldsymbol{D}}\right)$ & Type 4: $\left(\boldsymbol{\theta}_{L}^{\boldsymbol{A}}, \boldsymbol{\theta}_{L}^{\boldsymbol{D}}\right)$ \\
\hline
\end{tabular}

The admissions office (which we also refer to as $P 2$ ) suffers from the problem of asymmetric information. That is, P1 possesses a certain amount of private information - namely, what type of student they are (low ability/high ability, low demonstrated interest/high demonstrated interest, etc.). This information is unknown to P2 and can lead to the inefficient allocation of admission and scholarship awards.

A way P2 can gain access to this information is by screening P1 for their type. In our model, P2 has decided to use a standardized test and an interview as screening devices to probe P1's level of academic ability and demonstrated interest, respectively. The timing of this game is described below in Figure 1.

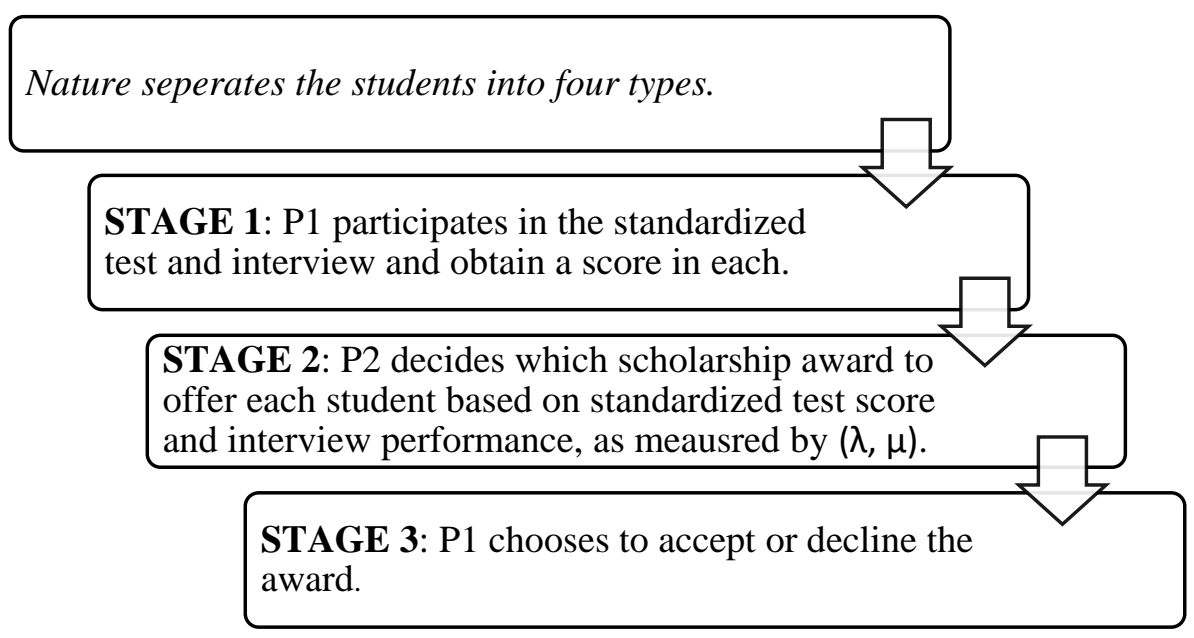

Figure 1. The timing of the admissions game

Nature draws a "type" for P1. Based on the type information, P1 participates in the standard test and interview, with the response recorded by a vector $(\lambda, \mu)$ where $\lambda \in(\underline{\lambda}, \bar{\lambda})$ represents the score of the standard test and $\mu \in$ $(\mu, \bar{\mu})$ represents the performance in the interview. Based on the scores $(\lambda, \mu)$, the admission office will decide to either "admit" or "reject" the applicant. If admitted, the admissions office can distribute a scholarship $S$ to P1. Based on the 
scholarship decision, P1 decides to accept or reject the offer vis-à-vis an alternative scholarship offer $\omega_{j}$ from other competitor schools, where $j$ denotes student type. Intuitively the admissions office wants to attract high-type students with a more generous scholarship.

We assume $\mathrm{P} 1$ will choose $(\lambda, \mu)$ to maximize the following utility function,

$$
U_{j}^{1}(\lambda, \mu)=\max \left\{\pi_{j}+S-c_{E}\left(\lambda, \theta^{A}\right)-c_{I}\left(\mu, \theta^{D}\right), \omega_{j}\right\}
$$

where $\pi_{j}$ is the intrinsic value of attending the university for type $\mathrm{j}$ students, $S$ is the amount of the scholarship received, and $c_{E}\left(c_{I}\right)$ is the irretrievable cost of preparing for the exam (interview). To be specific, we assume $\pi_{j}=\pi_{H}>0$, for $j=1,2$ (i.e., Type 1 and 2 students). That is, students with high demonstrated interest received a fixed positive utility $\pi_{H}$ from enrolling. In contrast, students with low demonstrated interest (i.e., Type 3 and 4) find no intrinsic value in attending the university: $\pi_{j}=0, j=3,4$. In addition, we assume the alternative offers satisfiy the following condition: $\omega_{1}>\omega_{3}>\omega_{2}>\omega_{4}>0$. This assumption reflects the idea that academic ability is valued higher than demonstrated interest.

The cost of preparing for the standardized test, $c_{E}\left(\lambda, \theta^{A}\right)$, depends not only on the anticipated test score $\lambda$ but also the level of the test taker's academic ability $\theta^{A} \in\left\{\theta_{H}^{A}, \theta_{L}^{A}\right\}$. Additional regularity assumptions are made on these cost functions:

1. Students receive zeros in exams and interviews when no effort is committed: $c_{I}\left(0, \theta^{D}\right)=c_{E}\left(0, \theta^{A}\right)=0$.

2. Both cost functions are assumed to be differentiable, strictly increasing with respect to their first argument, and strictly decreasing with respect to their second argument: $c_{1}>0$ and $c_{2}<0$, where $c_{k}$ denotes the partial derivative with respect to the k-th argument.

3. There exists $k, l>1$ such that $c_{E}\left(\lambda, \theta_{L}^{A}\right)=k c_{E}\left(\lambda, \theta_{H}^{A}\right)$ and $c_{I}\left(\mu, \theta_{L}^{D}\right)=l c_{I}\left(\mu, \theta_{H}^{D}\right)$ for all $\lambda$ and $\mu$.

The second assumption makes it so that obtaining a higher score incurs a larger cost on the students (i.e., $c_{1}>0$ ). In addition, students with low academic ability need to commit more effort to obtain the same score in the test relative to high ability students (i.e., $c_{2}<0$ ). The differential effort between the two types, as described in the third assumption, does not depend on the point scale. This third assumption can be relaxed at the expense of a more complex proof.

Recall that the objective of the admissions office is to make the students truthfully reveal their type. As will be established formally in the next section, the admissions office can use two different classes of scholarship, $S_{H}>S_{L}>$ 0 , to achieve this objective.

\section{Equilibrium}

The structure of the cost functions implies that low type students need to spend greater efforts to achieve the same score compared to the high type. As such, the high types are more likely to "signal" their credentials by achieving a certain score on the exam/interview, which the low type may not find it worthy to do so. It is common for the admissions office to develop a "threshold belief": that is, any $\lambda \geq \lambda^{*}$ makes P2 believe the student has high academic ability and any $\mu \geq \mu^{*}$ makes $\mathrm{P} 2$ believe the student has high desire and readiness.

It is important to note that P2's belief only changes at the threshold. That is, any marginal improvement in score on either side of the threshold will only lead to incremental cost on the student with no marginal benefit. Consequently, a rational high type student will score exactly $\lambda^{*}$ or $\mu^{*}$, whereas the low types find it reluctant to put forth any effort and hence achieve 0 . The mapping between observed score and implied type according to this "threshold-crossing" belief is reported below: in equilibrium, we expect the responses of the four types to be at the bottom-left corners of their respective quadrant.

A natural question to ask is: is it possible for the admissions office to set up a scoring system $\left(\lambda^{*}\right.$ and $\left.\mu^{*}\right)$ and scholarship award $S$ such that it is incentive-compatible for the four types of applicants to respond exactly this way? As shown below, the admissions office needs to set up two distinct scholarship, with amounts $S_{H}>S_{L}>0$. Once a set of restrictions are satisfied, a separating equilibrium can be induced in which each type of student takes a unique action according to the aforementioned belief. Consequently, their different responses to the standardized test and interview reveal their type. We first give a formal characterization of this equilibrium, and then discuss the restrictions needed to induce it. 


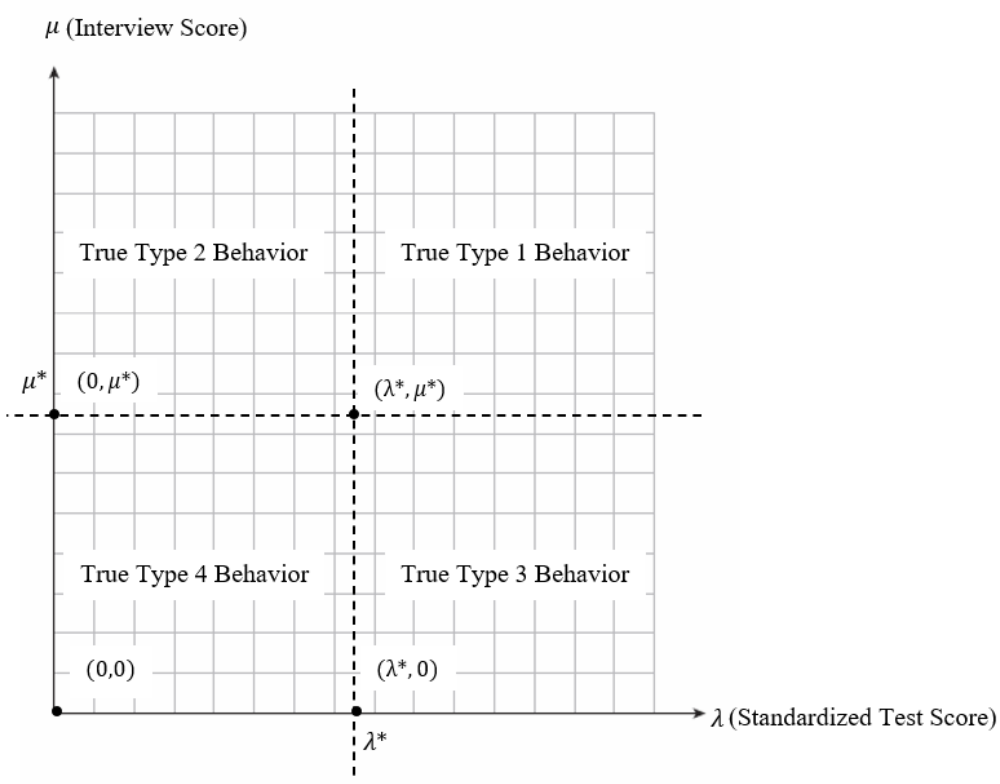

Figure 2. P2's Belief

Proposition 1 (Separating Equilibria): Once $\left(\lambda^{*}, \boldsymbol{\mu}^{*}\right)$ satisfy the five restrictions below, a separating equilibrium exists where Type 1 students respond with $\left(\lambda^{*}, \boldsymbol{\mu}^{*}\right)$ and accept $S_{H}$; Type 2 students choose $\left(\mathbf{0}, \boldsymbol{\mu}^{*}\right)$ and accept scholarship award 0; Type 3 students choose $\left(\lambda^{*}, \mathbf{0}\right)$ and accept $S_{L}$; and Type 4 students choose $(\mathbf{0}, \mathbf{0})$ and will be rejected.

(See Appendix for the proof)

The key takeaway of this equilibrium result is that the low-type P1 do not have an incentive to mimic the high type, so the combination of screening devices (i.e., exam + interview) unveils the private information of P1. In other words, the equilibrium causes P1 to truthfully reveal their types. Since ability is valued more than desire, the admissions office would only offer scholarship awards to those who succeed in the test to attract them. Furthermore, those of P1 with impressive interviews will receive a greater scholarship award for signaling the high desire to attend. We will go through the restrictions which induce the separating equilibrium below.

Restriction 1: $\operatorname{lc}\left(\mu^{*}, \theta_{H}^{D}\right)>S_{H}-S_{L}>c\left(\mu^{*}, \theta_{H}^{D}\right)$

That is, the difference between $S_{H}$ and $S_{L}$ must be exceeded by the cost of achieving $\mu^{*}$ for low-desire students and must exceed the cost of achieving $\mu^{*}$ for high-desire students. This restriction guarantees that Type 3 students will find $\left(\lambda^{*}, \mu^{*}\right)$ too costly to pursue. It will also guarantee that Type 1 students will accept $S_{H}$.

Restriction 2: $\quad \pi_{H}+S_{H}-\omega_{1}>c\left(\lambda^{*}, \theta_{H}^{A}\right)+c\left(\mu^{*}, \theta_{H}^{D}\right)$

That is, that the utility associated with $\left(\lambda^{*}, \mu^{*}\right)$ exceeds the alternative offer for Type 1 students. This guarantees that Type 1 students find the strategy associated with receiving $S_{H}$ to be more beneficial than their alternative offer.

Restriction 3: $\quad k c\left(\lambda^{*}, \theta_{H}^{A}\right)>S_{H}$

That is, the cost of achieving a score of $\lambda$ on the exam must exceed the value of $S_{H}$ for low-ability students. This restriction guarantees that Type 2 students will truthfully reveal their type, and not try to mimic the behavior of Type 1 students by signaling high academic ability.

Restriction 4: $\pi_{H}-\omega_{2}>c\left(\mu^{*}, \theta_{H}^{D}\right)$

That is, the difference between the intrinsic utility Type 2 students receive from attending the school and their alternative offer must exceed the cost they incur for achieving an interview score of $\mu$. This restriction guarantees that Type 2 students will find it in their best demonstrated interest the school without a scholarship.

Restriction 5: $S_{L}-c\left(\lambda^{*}, \theta_{H}^{A}\right)>\omega_{3}$

That is, the difference between $S_{L}$ and the cost of achieving an exam score $\lambda$ for high ability students must exceed the alternative offer of Type 3 students. This restriction guarantees that the utility Type 3 students obtain from accepting $S_{L}$ will be a more profitable alternative to $\omega_{3}$. 
To ensure the proposed separating is indeed implementable, we must ensure the five restrictions on $\lambda^{*}$ and $\mu^{*}$ are not mutually exclusive. That is, some $\left(\lambda^{*}, \mu^{*}\right)$ must satisfy all five restrictions simultaneously.

Proposition 2 (Existence of Equilibria): For any cost functions discussed in Section II.3, there exists some $\left(\lambda^{*}, \boldsymbol{\mu}^{*}\right)$ that satisfy the five restrictions above.

This proposition is proved in the Appendix, where we derive the bounds for $\left(\lambda^{*}, \boldsymbol{\mu}^{*}\right)$ from the restrictions and show the upper bounds exceed the lower bounds. Once the cost functions are known, the bounds can be solved in closed form. We provide an illustration below to show how it can be done.

\section{An Example}

In this section, we work out a simple example to serve as an antidote to the abstractness of the previous section and to solve for the bounds of $\lambda^{*}$ and $\mu^{*}$. These bounds are empirically relevant to the admissions office as they provide concrete guidance on how the evaluation standards on exams and interviews should be set.

Define $Z^{A}$ to be opportunity cost (measured as a dollar amount, say the forgone wages) on a student to improve his/her exam score by one point and $Z^{D}$ to be the opportunity cost on a student to improve his/her interview score by one point. Since it is less costly for students with high academic ability to prepare for the test, we have $Z_{L}^{A}>Z_{H}^{A}$. We define a student academic ability as the inverse of the opportunity cost: $\theta^{A}=\frac{1}{Z^{A}}$ so that $Z_{L}^{A}>Z_{H}^{A}$ implies $\theta_{H}^{A}>\theta_{L}^{A}$. In using parallel reasoning for the interview cost function, we define $\theta^{D}=\frac{1}{Z^{D}}$. To ensure consistency with the notation in Section II, we employ the following cost functions:

$$
\begin{aligned}
& c\left(\lambda, \theta^{A}\right)=\frac{\lambda}{\theta^{A}}=Z^{A} \lambda \\
& c\left(\mu, \theta^{D}\right)=\frac{\mu}{\theta^{D}}=Z^{D} \mu
\end{aligned}
$$

The assumed cost functions satisfy the three regularity conditions in Section II.3: $c\left(0, \theta^{D}\right)=c\left(0, \theta^{A}\right)=0$. With $k=\frac{\theta_{H}^{A}}{\theta_{L}^{A}}$, and $l=\frac{\theta_{H}^{D}}{\theta_{L}^{D}}, c\left(\lambda, \theta_{L}^{A}\right)=k c\left(\lambda, \theta_{H}^{A}\right)$ and $c\left(\mu, \theta_{L}^{D}\right)=l c\left(\mu, \theta_{H}^{D}\right)$ for all $\lambda$ and $\mu$.

We can now solve for the closed form of $\lambda^{*}$ and $\mu^{*}$ by using our restrictions, which induce a separating equilibrium. First, we rewrite the restrictions using the above cost functions:

$$
\begin{aligned}
& \text { 1. } \frac{\mu^{*} l}{\theta_{H}^{D}}>S_{H}-S_{L}>\frac{\mu^{*}}{\theta_{H}^{D}} \\
& \text { 2. } \pi_{H}+S_{H}-\omega_{1}>\frac{\lambda^{*}}{\theta_{H}^{A}}+\frac{\mu^{*}}{\theta_{H}^{D}} \\
& \text { 3. } \frac{\lambda^{*} k}{\theta_{H}^{A}}>S_{H} \\
& \text { 4. } \pi_{H}-\omega_{2}>\frac{\mu^{*}}{\theta_{H}^{D}} \\
& \text { 5. } S_{L}-\frac{\lambda^{*}}{\theta_{H}^{A}}>\omega_{3}
\end{aligned}
$$

We then isolate $\lambda^{*}$ and $\mu^{*}$ for each restriction.

1. $\frac{\mu^{*} l}{\theta_{H}^{D}}>S_{H}-S_{L}>\frac{\mu^{*}}{\theta_{H}^{D}} \Rightarrow \mu^{*} l>\theta_{H}^{D}\left(S_{H}-S_{L}\right)>\mu^{*} \Rightarrow \mu^{*}>\frac{\theta_{H}^{D}\left(S_{H}-S_{L}\right)}{l} \Rightarrow \boldsymbol{\mu}^{*}>\boldsymbol{\theta}_{\boldsymbol{L}}^{D}\left(\boldsymbol{S}_{\boldsymbol{H}}-\boldsymbol{S}_{\boldsymbol{L}}\right)$

2. We revisit Restriction 2 upon finding other potential upper bounds for $\lambda^{*}$ and $\mu^{*}$. 


$$
\begin{aligned}
& \text { 3. } \frac{\lambda^{*} k}{\theta_{H}^{A}}>S_{H} \Rightarrow \lambda * k>S_{H} \theta_{H}^{A} \Rightarrow \lambda *>\frac{S_{H} \theta_{H}^{A}}{k} \Rightarrow \lambda *>\boldsymbol{S}_{\boldsymbol{H}} \boldsymbol{\theta}_{\boldsymbol{L}}^{\boldsymbol{A}} \\
& \text { 4. } \pi_{H}-\omega_{2}>\frac{\mu^{*}}{\theta_{H}^{D}} \Rightarrow \boldsymbol{\theta}_{\boldsymbol{H}}^{\boldsymbol{D}}\left(\boldsymbol{\pi}_{\boldsymbol{H}}-\boldsymbol{\omega}_{2}\right)>\boldsymbol{\mu}^{*} \\
& \text { 5. } S_{L}-\frac{\lambda^{*}}{\theta_{H}^{A}}>\omega_{3} \Rightarrow \boldsymbol{\theta}_{\boldsymbol{H}}^{\boldsymbol{A}}\left(\boldsymbol{S}_{\boldsymbol{L}}-\boldsymbol{\omega}_{3}\right)>\lambda^{*}
\end{aligned}
$$

In revisiting Restriction 2, we determine if a tautology will be produced by substituting the upper bounds of $\lambda^{*}$ and $\mu^{*}$ found in Restrictions 5 and 4, respectively. If we are able to show that a tautology exists in this case, we eliminate Restriction 2 from consideration as the upper constraint it will pose to $\lambda^{*}$ and $\mu^{*}$ will exceed that of the alternatively presented upper constraints, rendering it unnecessary.

$$
\begin{gathered}
\pi_{H}+S_{H}-\omega_{1}>\frac{\theta_{H}^{A}\left(S_{L}-\omega_{3}\right)}{\theta_{H}^{A}}+\frac{\theta_{H}^{D}\left(\pi_{H}-\omega_{2}\right)}{\theta_{H}^{D}} \Rightarrow \pi_{H}+S_{H}-\omega_{1}>S_{L}-\omega_{3}+\pi_{H}-\omega_{2} \Rightarrow S_{H}-\omega_{1}>S_{L}-\omega_{3}-\omega_{2} \\
\Rightarrow S_{H}-\omega_{1}>\frac{1}{r}\left(S_{H}-\omega_{1}\right)-\omega_{2}
\end{gathered}
$$

A tautology is presented for any $r=\frac{S_{H}}{S_{L}}=\frac{\omega_{1}}{\omega_{3}}>1$. Therefore, our $\lambda^{*}$ and $\mu^{*}$ are determined completely by constraints $1,3,4$, and 5. Substituting $\theta^{A}=\frac{1}{Z^{A}}$ and $\theta^{D}=\frac{1}{Z^{D}}$, the admissible range of $\lambda^{*}$ and $\mu^{*}$ can be succinctly presented as

$$
\begin{aligned}
& \frac{\left(S_{L}-\omega_{3}\right)}{Z_{H}^{A}}>\lambda *>\frac{S_{H}}{Z_{L}^{A}} \\
& \frac{\left(\pi_{H}-\omega_{2}\right)}{Z_{H}^{D}}>\mu^{*}>\frac{\left(S_{H}-S_{L}\right)}{Z_{L}^{D}}
\end{aligned}
$$

To gain additional insight, we can multiply (a) by $Z_{L}^{A}$ and we get $\lambda * Z_{L}^{A}>S_{H}$. This implies that for students with low academic ability (Type 2, for example), the economic cost of obtaining the "passing score" on the exam must exceed the high scholarship reward. As such, such a constraint effectively forestalls them from mimicking the high ability students (Type 1). Similarly, in multiplying (a) by $Z_{H}^{A}$, we get $S_{L}-\omega_{3}>\lambda * Z_{H}^{A}$. This ensures that the economic cost of preparing for and completing the test is low enough for Type 3 students (high ability and low desire and readiness) such that the net benefit of receiving $S_{L}$ still exceeds the alternative offer. We show the cost associated with the standardized test as a function of $\lambda$ in the graph below to present the permissible range of $\lambda^{*}$ graphically.

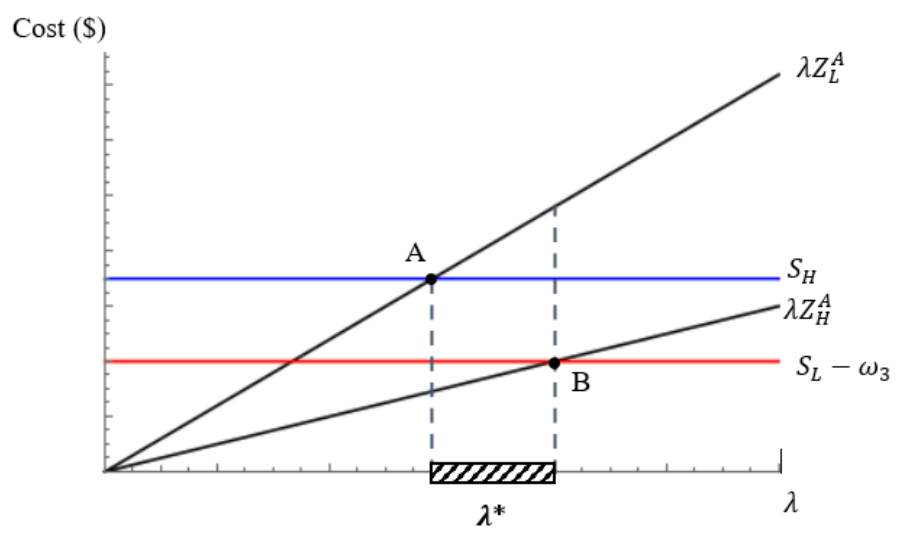

Figure 3. $\lambda$ graphed as a function of $Z$ 
Point $A$ serves as a break-even point for Type 2 students, where the cost of obtaining the high scholarship equals the value of the high scholarship, leaving them indifferent to the imitation of Type 1 behavior at this value of $\lambda$. Point $B$ serves as a break-even point for Type 3 students, where the cost of obtaining the low scholarship, which is $\lambda Z_{H}^{A}+\omega_{3}$, equals the value of the low scholarship. At point $B$, Type 3 students find themselves indifferent to the imitation of Type 4 behavior. Thus, $\lambda^{*}$ excludes the $\lambda$ values of points $A$ and $B$ but includes all of the values of $\lambda$ in between.

Turning to constraint (b), we first multiply $Z_{L}^{D}$ and get $\mu * Z_{L}^{D}>S_{H}-S_{L}$. This ensures that Type 3 students do not bother to mimic Type 1 students as the scholarship differential is no greater than the additional cost of preparing for the interview. Lastly, we multiply (b) by $Z_{H}^{D}$ and we get $\mu * Z_{H}^{D}<\pi_{H}-\omega_{2}$. For Type 2 students (high desire and readiness and low ability), it is still cost-effective for them to prepare for and pass the interview stage so they can attend the university and obtain $\pi_{H}$.

We show the cost associated with the interview as a function of $\mu$ in the graphs below. We simultaneously represent the range of $\mu^{*}$. Because it is not certain whether $S_{H}-S_{L}$ is greater than, less than or equal to $\pi_{H}-\omega_{2}$, we provide a graph for each of the cases.

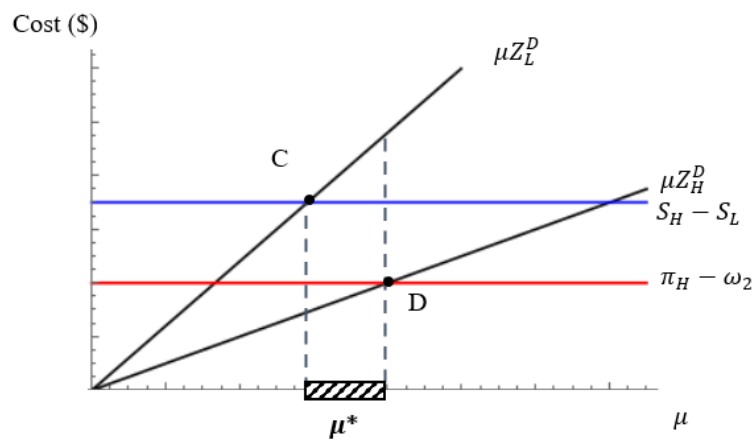

Figure 4. $\mu$ graphed as a function of $Z$ where $S_{H}-S_{L}>\pi_{H}-\omega_{2}$.

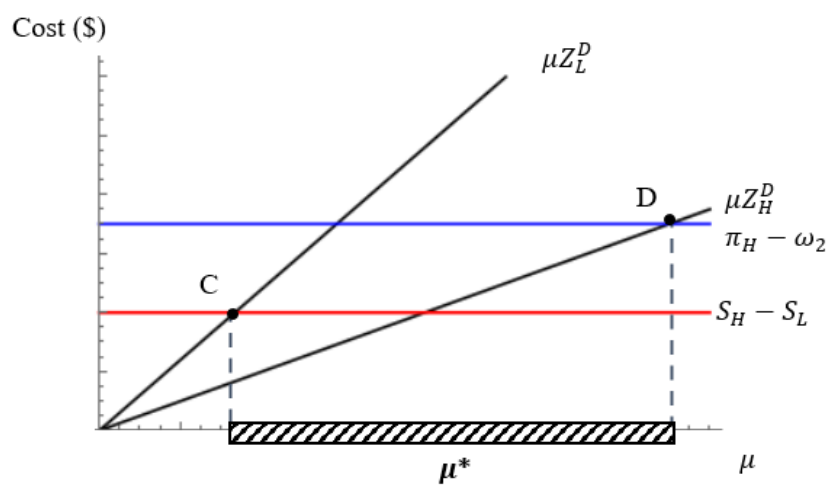

Figure 5. $\mu$ graphed as a function of $Z$ where $S_{H}-S_{L}<\pi_{H}-\omega_{2}$. 


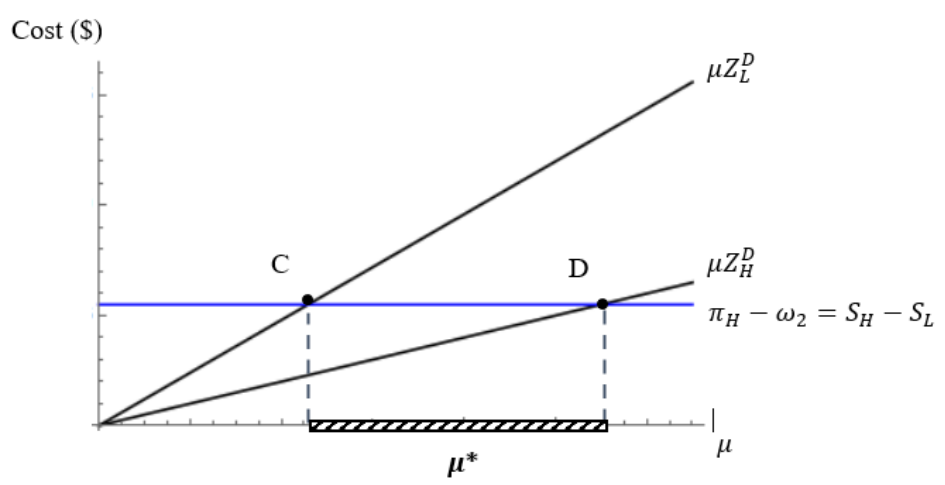

Figure 6. $\mu$ graphed as a function of $Z$ where $S_{H}-S_{L}=\pi_{H}-\omega_{2}$.

In each case, point $C$ serves as a break-even point for Type 3 students, where the differential between the two scholarship awards is equal to the cost of passing the interview task, which leaves them indifferent to imitating Type 1 students by achieving $\mu^{*}$. Point $D$ serves as a break-even point for Type 2 students, where the differential between the intrinsic value they receive from merely attending the school and their alternative offer is equal to the cost of achieving $\mu$. At point $D$, Type 2 students find themselves indifferent to the imitation of Type 4 behavior. Thus, $\mu^{*}$ excludes the $\mu$ values of points $C$ and $D$ but includes all of the values of $\mu$ in between.

In summary, constraint (a) ensures that only Type 3 (and not Type 2) will make the effort in passing the exam and constraint (b) ensures that only Type 2 (and not Type 3) will make the effort in passing the interview. As a result, both types are incentivized to signal their ability/interests appropriately in the test/interview stage, respectively.

\section{Comparative Statics}

In our model, the range of $\lambda^{*}$ and $\mu^{*}$ are endogenously determined by exogenous variables $Z, S, \omega$, and $\pi$. Using our findings, we can answer several questions regarding comparative statics, the manners in which $\lambda^{*}$ and $\mu^{*}$ are affected by changes in these exogenous variables.

In the case of both $\lambda$ and $\mu, Z_{H}$ and $Z_{L}$ affect the sizes of the sets $\lambda^{*}$ and $\mu^{*}$. Specifically, if the differential between $Z_{H}$ and $Z_{L}$ increases, then the sizes - that is, the ranges of scores - of $\lambda^{*}$ and $\mu^{*}$ widen (for $\mu^{*}$, this phenomenon occurs for all three cases covered in Figures 4-6). Thus, if the cost differential for completing each application task increases between the high and low type students, then the lower bounds for $\lambda^{*}$ and $\mu^{*}$ decrease and the upper bounds increase and vice versa. This relationship is due to the fact that in the functions $\lambda Z^{A}$ and $\mu Z^{D}, Z$ acts as the slope of the function, and when we, for instance, increase the differential between $Z_{H}^{A}$ and $Z_{L}^{A}$, then $\lambda Z_{H}^{A}$ and $\lambda Z_{L}^{A}$ diverge more dramatically and the break-even points move away from each other, leaving us with a larger set for $\lambda^{*}$ (this same phenomenon occurs for $\mu^{*}$ ).

In our analysis, we assume the value $\frac{s_{H}}{S_{L}}$ to be a constant $r$, and in the name of consistency, we cannot consider a movement in one without simultaneously moving the other proportionately. In the case of $\lambda$, an increase in one and a proportionate increase in the other will drive up both the lower and upper bounds of $\lambda^{*}$. It is unclear at this point how this change will affect the size of the set of $\lambda^{*}$. In the case of $\mu$, an increase in one and a proportionate increase in the other will move the lower bound of $\mu^{*}$ in the positive direction laterally. This makes the standards for passing the interview process more difficult for the student. In this case, we can also deduce that the range of $\mu^{*}$ will contract.

Turning to $\omega$, when $\omega_{2}$ increases the upper bound for $\mu^{*}$ decreases. This indicates that when the alternative offer for Type 2 students increases, fewer interview scores could be considered by the admissions office as the passing score. Alternatively, when $\omega_{3}$ increases the upper bound for $\lambda^{*}$ increases. That is, when the alternative offer for Type 3 
students increases, higher scores for the standardized test could be considered by the admissions office as the passing score.

Lastly, when $\pi_{H}$ increases the upper bound for $\mu^{*}$ increases. Thus, $\pi_{H}$ and the direction of change for the upper extremity for $\mu^{*}$ are positively related.

\section{Empirical Implementation/Implication}

In this section, we discuss to what extent the theoretical model is consistent with the current status of college admission in the U.S. as well as what policy implications can this theoretical framework offer.

Admittedly, the most suitable way of validating the theoretical model is to collaborate with university admission office to conduct experiment. Given the amount of time and resource that such experiments can potentially take, we would leave them as future research work. Nevertheless, we would like to present some empirical evidence from other studies to support our model prediction.

First of all, the results of this study confirmed research regarding high school GPA and other pre-college variables as a successful significant positive predictor for the admission outcome (Astin, 1997; Hoffman \& Lowitzki, 2005; Schwartz \& Washington, 2002; Ting, 1998; Wolfe \& Johnson, 1995). After all, if applicants are incentivized to cram for standardized tests and interviews to game the admission system (Bound et al., 2009), the admission office ought to use pre-college variables to screen applicants. In a recent study, by analyzing data from 192 institutions, Sawyer (2013) suggests that high school GPA is more useful than admission test scores in situations involving low selectivity in admissions and minimal to average academic performance in college.

Another important conclusion of the model is that scholarship awards can be used as effective means to increase enrollment rate. However, the admission criteria must be adjusted according to the scholarship amount to ensure the separating equilibrium outcome (i.e., as specified in equations (a)-(b)). The complexity of the proposed mechanism might, to some extent, explain the mixed empirical findings in regards to the enrollment effect on scholarship. In Bartik, Hershbein and Lachowska (2015), the authors estimate the effects on postsecondary education outcomes of the Kalamazoo Promise, a generous place-based college scholarship. Using difference-in-differences, the authors find the Promise significantly increases college enrollment. In contrast, Bozick, Gonzalez and Engberg (2015), using admission data from the Pittsburgh Promise scholarship program, showed that the scholarship had no direct effect on the overall rate of college enrollment.

\section{Conclusion}

We have employed our methodology and induced a response from P1 that corresponds to true type behavior. Recall that this was accomplished by applying the separating equilibrium, which is designed specifically to separate the students into their true types. The strategy we propose consists of manipulating the scoring thresholds for the exam and interview in such a fashion that meeting said thresholds becomes too costly for low-type students.

We show in Section VI of this paper that there exist a set of both $\lambda$ and $\mu$ that satisfy each of the restrictions for all $c\left(\lambda, \theta^{A}\right)$ and $c\left(\mu, \theta^{D}\right)$. Thus, in our game, it is always possible to induce the equilibrium. There are economic consequences for deviating from the equilibrium.

1) Violating Restriction 1 would either provide an incentive for Type 3 students to mimic Type 1 behavior, fail to provide enough of an incentive for Type 1 students to accept P2's offer of $S_{H}$, or both.

2) Violating Restriction 2 would fail to provide enough of an incentive for Type 1 students to engage in true Type 1 behavior.

3) Violating Restriction 3 would fail to take away any incentive for Type 2 students to mimic Type 1 behavior.

4) Violating Restriction 4 would fail to provide enough of an incentive for Type 2 students to enroll without scholarship.

5) Violating Restriction 5 would fail to provide enough of an incentive for Type 3 students to engage in true Type 3 behavior. 


\section{Acknowledgements}

We thank the editor and two reviewers for their constructive comments. Joe Rekus would like to thank the Office of Undergraduate Research and Creative Activity (OURCA) at Christopher Newport University for funding this research. All errors remain ours.

\section{References}

Astin, A. W. (1997). How "good" is your institution's retention rate?. Research in higher education, 38(6), 647-658. https://doi.org/10.1023/A:1024903702810

Ball, R., Robin, A., \& Wu, J. S. (2003). Incentives versus standards: properties of accounting income in four East Asian countries. Journal of accounting and economics, 36(1-3), 235-270. https://doi.org/10.1016/j.jacceco.2003.10.003

Bartik, T., Hershbein, B., \& Lachowska, M. (2015). The effects of the Kalamazoo Promise scholarship on college enrollment, persistence, and completion. Persistence, and Completion (June 29, 2015). https://doi.org/10.2139/ssrn.2624727

Bound, J., Brad, H., \& Bridget, T. L. (2009). Playing the admissions game: Student reactions to increasing college competition. Journal of Economic Perspectives, 23(4), 119-46. https://doi.org/10.1257/jep.23.4.119

Bozick, R., Gonzalez, G., \& Engberg, J. (2015). Using a merit-based scholarship program to increase rates of college enrollment in an urban school district: The case of the Pittsburgh Promise. Journal of Student Financial Aid, 45(2), 2.

Bushman, R. M., \& Smith, A. J. (2001). Financial accounting information and corporate governance. Journal of accounting and Economics, 32(1-3), 237-333. https://doi.org/10.1016/S0165-4101(01)00027-1

Clinedinst, M. (2019). 2019 State of College Admission. National Association for College Admission Counseling.

Dearden, J. A., Li, S. H., Chad, D. M., \& Yang, M. Z. (2017). Demonstrated interest: Signaling behavior in college admissions. Contemporary Economic Policy, 35(4), 630-657. https://doi.org/10.1111/coep.12216

Farber, D. B. (2005). Restoring trust after fraud: Does corporate governance matter? The Accounting Review, 80(2), 539-561. https://doi.org/10.2308/accr.2005.80.2.539

Hoffman, J. L., \& Lowitzki, K. E. (2005). Predicting college success with high school grades and test scores: Limitations for minority students. The review of higher education,28(4), 455-474. https://doi.org/10.1353/rhe.2005.0042

Rothschild, M., \& Stiglitz, J. (1976). Equilibrium in Competitive Insurance Markets: An Essay on the Economics of Imperfect Information. The Quarterly Journal of Economics, 90(4), 629-649. https://doi.org/10.2307/1885326

Sawyer, R. (2013). Beyond correlations: Usefulness of high school GPA and test scores in making college admissions decisions. Applied measurement in education, 26(2), 89-112. https://doi.org/10.1080/08957347.2013.765433

Schwartz, R. A., \& Washington, C. M. (2002). Predicting academic performance and retention among African American freshmen men. NASPA Journal, 39(4), 354-370. https://doi.org/10.2202/1949-6605.1178

Spence, M. (1973). Job Market Signaling. The Quarterly Journal of Economics, 87(3), 355-374. https://doi.org/10.2307/1882010

Ting, S. M. (1998). Predicting First-Year Grades and Academic Progress of College Students of First-Generation and Low-Income Families. Journal of College Admission, 158, 14-23.

Wolfe, R. N., \& Johnson, S. D. (1995). Personality as a predictor of college performance. Educational and psychological measurement, 55(2), 177-185. https://doi.org/10.1177/0013164495055002002 


\section{Appendix}

In this section, we will prove that Proposition 1 is the result of the game if all of the restrictions are assumed. Thus, we will show that the five restrictions cause each type to act according to the proposition. Proposition 2 argues the existence of $\lambda^{*}$ and $\mu^{*}$ for any and all $c\left(\lambda, \theta^{A}\right)$ and $c\left(\mu, \theta^{D}\right)$ and we show this as well. Note that $c$ is a cost function of the form $c(x, y)$ and is positively related with $x$. Thus, for any $x_{1}>x_{2}, c\left(x_{1}, y\right)>c\left(x_{2}, y\right)$.

Recall:

Proposition 1: Once $\left(\lambda^{*}, \mu^{*}\right)$ satisfy the five restrictions below, a separating equilibrium exists where Type 1 students respond with $\left(\lambda^{*}, \boldsymbol{\mu}^{*}\right)$ and accept $S_{H}$; Type 2 students choose $\left(\mathbf{0}, \boldsymbol{\mu}^{*}\right)$ and accept scholarship award 0; Type 3 students choose $\left(\lambda^{*}, \mathbf{0}\right)$ and accept $S_{L}$; and Type 4 students choose $(\mathbf{0}, \mathbf{0})$ and will be rejected.

Where the restrictions - numbered 1 through 5 - are:

1. $\operatorname{lc}\left(\mu, \theta_{H}^{D}\right)>S_{H}-S_{L}>c\left(\mu, \theta_{H}^{D}\right)$

2. $\pi_{H}+S_{H}-\omega_{1}>c\left(\lambda, \theta_{H}^{A}\right)+c\left(\mu, \theta_{H}^{D}\right)$

3. $k c\left(\lambda, \theta_{H}^{A}\right)>S_{H}$

4. $\pi_{H}-\omega_{2}>c\left(\mu, \theta_{H}^{D}\right)$

5. $S_{L}-c\left(\lambda, \theta_{H}^{A}\right)>\omega_{3}$

Proposition 1 claims that each type of student takes a unique strategy. It also claims that each type of student makes a unique decision about their scholarship offer. To prove that under these restrictions no student will find an incentive to mimic another types' true behavior, we show that the utility they obtain from pursuing their true behavior, prescribed to them in Proposition 1, exceeds the utility they would obtain from such imitation.

Consider Table 6 , where $U_{j}^{i}$ is the utility of signaling type $i$ for any student type $j$.

Table 6. Utility from strategy.

\begin{tabular}{|l|c|c|c|c|}
\hline \multicolumn{1}{|c|}{$U_{j}^{i}$} & $\left(\lambda^{*}, \mu^{*}\right)$ & $\left(0, \mu^{*}\right)$ & $\left(\lambda^{*}, 0\right)$ & $(0,0)$ \\
\hline Type 1 & $U_{1}^{1}$ & $U_{1}^{2}$ & $U_{1}^{3}$ & $U_{1}^{4}$ \\
\hline Type 2 & $U_{2}^{1}$ & $U_{2}^{2}$ & $U_{2}^{3}$ & $U_{2}^{4}$ \\
\hline Type 3 & $U_{3}^{1}$ & $U_{3}^{2}$ & $U_{3}^{3}$ & $U_{3}^{4}$ \\
\hline Type 4 & $U_{4}^{1}$ & $U_{4}^{2}$ & $U_{4}^{3}$ & $U_{4}^{4}$ \\
\hline
\end{tabular}

We must show that in assuming the above restrictions, for each $j U_{j}^{i}$ where $i=j$ exceeds every $U_{j}^{i}$ where $i \neq j$. The utility of being rejected from the university is the student's alternative offer.

For Type 1 students, we show that:

- $\quad$ Restriction 5 gives us $S_{L}>c\left(\lambda^{*}, \theta_{H}^{A}\right)+\omega_{3} \Rightarrow S_{L}>c\left(\lambda^{*}, \theta_{H}^{A}\right) \Rightarrow S_{H}-c\left(\lambda^{*}, \theta_{H}^{A}\right)>0 \Rightarrow \pi_{H}+S_{H}-$ $c\left(\lambda^{*}, \theta_{H}^{A}\right)-c\left(\mu^{*}, \theta_{H}^{D}\right)>\pi_{H}-c\left(\mu^{*}, \theta_{H}^{D}\right) \Rightarrow U_{1}^{1}>U_{1}^{2}$.

- Restriction 1 gives us $S_{H}-S_{L}>c\left(\mu^{*}, \theta_{H}^{D}\right) \Rightarrow S_{H}-c\left(\mu^{*}, \theta_{H}^{D}\right)>S_{L} \Rightarrow \pi_{H}+S_{H}-c\left(\lambda^{*}, \theta_{H}^{A}\right)-$ $c\left(\mu^{*}, \theta_{H}^{D}\right)>\pi_{H}+S_{L}-c\left(\lambda^{*}, \theta_{H}^{A}\right) \Rightarrow U_{1}^{1}>U_{1}^{3}$.

- Restriction 2 gives us $\pi_{H}+S_{H}-\omega_{1}>c\left(\lambda^{*}, \theta_{H}^{A}\right)+c\left(\mu^{*}, \theta_{H}^{D}\right) \Rightarrow \pi_{H}+S_{H}-c\left(\lambda^{*}, \theta_{H}^{A}\right)-c\left(\mu^{*}, \theta_{H}^{D}\right)>\omega_{1} \Rightarrow U_{1}^{1}>U_{1}^{4}$.

For Type 2 students, we show that:

- Restriction 3 gives us $k c\left(\lambda^{*}, \theta_{H}^{A}\right)>S_{H} \Rightarrow \pi_{H}-c\left(\mu^{*}, \theta_{H}^{D}\right)>\pi_{H}+S_{H}-k c\left(\lambda^{*}, \theta_{H}^{A}\right)-c\left(\mu^{*}, \theta_{H}^{D}\right) \Rightarrow$ $U_{2}^{2}>U_{2}^{1}$.

- $\quad$ Restrictions 1 and 3 give us $S_{H}-\left(\mu^{*}, \theta_{H}^{D}\right)>S_{L}$ and $k c\left(\lambda^{*}, \theta_{H}^{A}\right)>S_{H}$, ergo $k c\left(\lambda^{*}, \theta_{H}^{A}\right)-$ $\left(\mu^{*}, \theta_{H}^{D}\right)>S_{L} \Rightarrow \pi_{H}-c\left(\mu^{*}, \theta_{H}^{D}\right)>\pi_{H}+S_{L}-k c\left(\lambda^{*}, \theta_{H}^{A}\right) \Rightarrow U_{2}^{2}>U_{2}^{3}$.

- Restriction 3 gives us $\pi_{H}-\omega_{2}>c\left(\mu^{*}, \theta_{H}^{D}\right) \Rightarrow \pi_{H}-c\left(\mu^{*}, \theta_{H}^{D}\right)>\omega_{2} \Rightarrow U_{2}^{2}>U_{2}^{4}$.

For Type 3 students, we show that: 
- $\quad$ Restriction 1 gives us $l c\left(\mu^{*}, \theta_{H}^{D}\right)>S_{H}-S_{L} \Rightarrow S_{L}>S_{H}-l c\left(\mu^{*}, \theta_{H}^{D}\right) \Rightarrow S_{L}-c\left(\lambda^{*}, \theta_{H}^{A}\right)>S_{H}-$ $c\left(\lambda^{*}, \theta_{H}^{A}\right)-l c\left(\mu^{*}, \theta_{H}^{D}\right) \Rightarrow U_{3}^{3}>U_{3}^{1}$.

- Restriction 5 gives us $S_{L}-\omega_{3}>c\left(\lambda^{*}, \theta_{H}^{A}\right) \Rightarrow S_{L}>c\left(\lambda^{*}, \theta_{H}^{A}\right) \Rightarrow S_{L}+\operatorname{lc}\left(\mu^{*}, \theta_{H}^{D}\right)>c\left(\lambda^{*}, \theta_{H}^{A}\right) \Rightarrow$ $S_{L}-c\left(\lambda^{*}, \theta_{H}^{A}\right)>-l c\left(\mu^{*}, \theta_{H}^{D}\right) \Rightarrow U_{3}^{3}>U_{3}^{2}$.

- Restriction 5 gives us $S_{L}-c\left(\lambda^{*}, \theta_{H}^{A}\right)>\omega_{3} \Rightarrow U_{3}^{3}>U_{3}^{4}$.

For Type 4 students, we show that:

- Restriction 3 gives us $k c\left(\lambda^{*}, \theta_{H}^{A}\right)>S_{H} \Rightarrow \omega_{4}+k c\left(\lambda^{*}, \theta_{H}^{A}\right)+l c\left(\mu^{*}, \theta_{H}^{D}\right)>S_{H} \Rightarrow \omega_{4}>S_{H}-$ $k c\left(\lambda^{*}, \theta_{H}^{A}\right)-l c\left(\mu^{*}, \theta_{H}^{D}\right) \Rightarrow U_{4}^{4}>U_{4}^{1}$.

- The statement $\omega_{4}>-l c\left(\mu^{*}, \theta_{H}^{D}\right)$ true for any combination of $\omega_{4}, l$, and $c\left(\mu^{*}, \theta_{H}^{D}\right)$ as all are greater than zero. Thus, $\omega_{4}>-l c\left(\mu^{*}, \theta_{H}^{D}\right) \Rightarrow U_{4}^{4}>U_{4}^{2}$.

- Restriction 3 gives us $k c\left(\lambda^{*}, \theta_{H}^{A}\right)>S_{L} \Rightarrow \omega_{4}+k c\left(\lambda^{*}, \theta_{H}^{A}\right)>S_{L} \Rightarrow \omega_{4}>S_{L}-k c\left(\lambda^{*}, \theta_{H}^{A}\right) \Rightarrow U_{4}^{4}>$ $U_{4}^{3}$.

We have thus shown that for each $j U_{j}^{i}$ where $i=j$ exceeds every $U_{j}^{i}$ where $i \neq j$. Therefore, Type 1 students respond with $\left(\lambda^{*}, \boldsymbol{\mu}^{*}\right)$ and accept $S_{H}$; Type 2 students choose $\left(\mathbf{0}, \boldsymbol{\mu}^{*}\right)$ and accept scholarship award 0; Type 3 students choose $\left(\lambda^{*}, \mathbf{0}\right)$ and accept $S_{L}$; and Type 4 students choose $(\mathbf{0}, \mathbf{0})$ and will be rejected.

Recall:

Proposition 2: For any cost functions discussed in Section II.3, there exists some $\left(\lambda^{*}, \boldsymbol{\mu}^{*}\right)$ that satisfy the five restrictions above.

Using the restrictions, we can invert the cost functions to give us potential upper bounds and lower bounds of $\lambda^{*}$ and $\mu^{*}$. In inverting Restrictions $1,3,4$, and 5 , we obtain the five following constraints:

i. $\mu^{*}>c^{-1}\left(\frac{S_{H}-S_{L}}{l}, \theta_{H}^{D}\right)$, a potential lower bound for $\mu^{*}$.

ii. $\quad c^{-1}\left(S_{H}-S_{L}, \theta_{H}^{D}\right)>\mu^{*}$, a potential upper bound for $\mu^{*}$.

iii. $\quad \lambda^{*}>c^{-1}\left(\frac{S_{H}}{k}, \theta_{H}^{A}\right)$, a potential lower bound for $\lambda^{*}$.

iv. $\quad c^{-1}\left(\pi_{H}-\omega_{2}, \theta_{H}^{D}\right)>\mu^{*}$, a potential upper bound for $\mu^{*}$.

v. $\quad c^{-1}\left(S_{L}-\omega_{3}, \theta_{H}^{A}\right)>\lambda^{*}$, a potential upper bound for $\lambda^{*}$.

In substituting the potential upper bounds for $\lambda^{*}$ and $\mu^{*}$ for Restriction 2, we find

$$
\begin{gathered}
\pi_{H}+S_{H}-\omega_{1}>c\left(c^{-1}\left(S_{L}-\omega_{3}, \theta_{H}^{A}\right), \theta_{H}^{A}\right)+c\left(c^{-1}\left(\pi_{2}-\omega_{2}, \theta_{H}^{D}\right), \theta_{H}^{D}\right) \Rightarrow \\
\pi_{H}+S_{H}-\omega_{1}>S_{L}-\omega_{3}+\pi_{2}-\omega_{2} \Rightarrow \\
S_{H}-\omega_{1}>S_{L}-\omega_{3}-\omega_{2} \Rightarrow \\
S_{H}-\omega_{1}>\frac{1}{r}\left(S_{H}-\omega_{1}\right)-\omega_{2} \\
\text { and } \\
\pi_{H}+S_{H}-\omega_{1}>c\left(c^{-1}\left(S_{L}-\omega_{3}, \theta_{H}^{A}\right), \theta_{H}^{A}\right)+c\left(c^{-1}\left(S_{H}-S_{L}, \theta_{H}^{D}\right), \theta_{H}^{D}\right) \Rightarrow \\
\pi_{H}+S_{H}-\omega_{1}>S_{L}-\omega_{3}+S_{H}-S_{L} \Rightarrow \\
\pi_{H}+S_{H}-\omega_{1}>S_{H}-\omega_{3} \Rightarrow \\
\pi_{H}-\omega_{1}>-\omega_{3} \Rightarrow \\
\pi_{H}+\omega_{3}>\omega_{1} \Rightarrow \\
\pi_{H}>\omega_{1}-\frac{\omega_{1}}{r} \Rightarrow \\
r\left(\pi_{H}-\omega_{1}\right)>-\omega_{1} \Rightarrow
\end{gathered}
$$




$$
\begin{gathered}
r>\frac{-\omega_{1}}{\pi_{H}-\omega_{1}} \Rightarrow \\
r>\frac{-\omega_{1}}{\pi_{H}-\omega_{1}}>1
\end{gathered}
$$

The first result is a tautology. The second result shows that there is no contradiction as $r$ is assumed to be greater than 1. This tells us that the maximum values of $\lambda^{*}$ and $\mu^{*}$ provided by Constraints ii, iv, and v satisfy Restriction 2 . Thus, Restriction 2 does not provide a minimum upper bound on either $\lambda^{*}$ or $\mu^{*}$.

To prove the existence of $\lambda^{*}$ and $\mu^{*}$, we show that the upper bounds of each exceed the lower bounds of each.

Restriction 5 gives us $S_{L}-\omega_{3}>c\left(\lambda^{*}, \theta_{H}^{A}\right)$ and Restriction 3 gives us $c\left(\lambda^{*}, \theta_{H}^{A}\right)>\frac{S_{H}}{k}$. Thus, $S_{L}-\omega_{3}>\frac{S_{H}}{k} \Rightarrow$ $c^{-1}\left(S_{L}-\omega_{3}, \theta_{H}^{A}\right)>c^{-1}\left(\frac{S_{H}}{k}, \theta_{H}^{A}\right) \Rightarrow c^{-1}\left(S_{L}-\omega_{3}, \theta_{H}^{A}\right)>\lambda^{*}>c^{-1}\left(\frac{S_{H}}{k}, \theta_{H}^{A}\right)$. Thus, $\lambda^{*}$ exists.

$S_{H}-S_{L}>\frac{S_{H}-S_{L}}{l}$ is true as $l>1$. Thus, $c^{-1}\left(S_{H}-S_{L}, \theta_{H}^{D}\right)>c^{-1}\left(\frac{S_{H}-S_{L}}{l}, \theta_{H}^{D}\right) \Rightarrow c^{-1}\left(S_{H}-S_{L}, \theta_{H}^{D}\right)>\mu^{*}>$ $c^{-1}\left(\frac{S_{H}-S_{L}}{l}, \theta_{H}^{D}\right)$. Restriction 4 gives us $\pi_{H}-\omega_{2}>\left(\mu^{*}, \theta_{H}^{D}\right)$ and Restriction 1 gives us $c\left(\mu^{*}, \theta_{H}^{D}\right)>\frac{S_{H}-S_{L}}{l}$. Thus, $\pi_{H}-\omega_{2}>\frac{S_{H}-S_{L}}{l} \Rightarrow c^{-1}\left(\pi_{H}-\omega_{2}, \theta_{H}^{D}\right)>c^{-1}\left(\frac{S_{H}-S_{L}}{l}, \theta_{H}^{D}\right) \Rightarrow c^{-1}\left(\pi_{H}-\omega_{2}, \theta_{H}^{D}\right)>\mu^{*}>c^{-1}\left(\frac{S_{H}-S_{L}}{l}, \theta_{H}^{D}\right)$. Thus, $\boldsymbol{\mu}^{*}$ exists.

\section{Copyrights}

Copyright for this article is retained by the author(s), with first publication rights granted to the journal.

This is an open-access article distributed under the terms and conditions of the Creative Commons Attribution license which permits unrestricted use, distribution, and reproduction in any medium, provided the original work is properly cited. 